\title{
Hepatitis B Virus in Pregnancy
}

\author{
Tram T. Tran, M.D.
}

Chronic hepatitis B virus (HBV) infection remains an important worldwide cause of chronic liver disease, which may lead to the development of cirrhosis, decompensated liver disease, and/or hepatocellular carcinoma. It is estimated that 2 billion people worldwide have been infected with HBV, 350 million of whom are chronically infected, with 50 million new cases diagnosed annually, despite the availability of an effective and safe vaccination. In the United States, the overall prevalence of HBV infection is 1.25 million, ${ }^{1}$ which may not account for some high-risk groups that would increase this estimate to 2 million. ${ }^{2}$

The prevalence of HBV infection varies greatly in different regions of the world, with rates of detectable hepatitis $B$ surface antigen (HBsAg) ranging from 15\% in highly endemic areas such as sub-Saharan Africa, Asia (excluding Japan), the Pacific Basin, parts of the Middle East, and the Amazon Basin to less than $2 \%$ in North America and western Europe. In areas of low endemicity, immigration patterns contribute to local variability in prevalence rates of HBV infection, particularly in communities with a large number of immigrants from highly endemic areas, many of whom may be women of childbearing age.

HBV is effectively transmitted via percutaneous or mucosal exposure to infectious blood or bodily fluids that contain blood. In regions of high endemicity, perinatal transmission from an infected mother to her newborn child remains an important mode of HBV infection, although there is also a substantial contribution from early horizontal transmission between infected family members or other individuals. For an infant whose mother is chronically infected and has serologic evidence of active HBV replication based on detection of hepatitis $\mathrm{B}$ e antigen ( $\mathrm{HBeAg}$ ), the risk for chronic HBV infection is high and ranges from $70 \%$ to $90 \%$ by the age of 6 months in the absence of passive immunoprophylaxis with hepatitis B immunoglobulin (HBIg) and hepatitis B vaccination. Children born to HBeAg-negative mothers have a lower risk of infection that ranges from $10 \%$ to $40 \%$, although most of those who do get infected will also develop chronic infection. ${ }^{3}$ Standard passive-active immunoprophylaxis with HBIg and hepatitis B vaccination administered immediately after birth to infants of HBsAg-positive mothers significantly reduces the risk of $\mathrm{HBV}$ transmission. However, despite these measures, $5 \%$ to $10 \%$ of infants born to HBeAg-positive mothers subsequently become HBsAg-positive. This infection rate may be related to high levels of maternal viremia, intrauterine infection, or HBV mutation of the surface protein. $^{4-6}$

\section{Risk of Transmission}

The likelihood of chronic infection in a newly infected individual is highly dependent on age at the time of infection. Children infected perinatally have a much higher risk of chronicity, with $90 \%$ of infants progressing to chronic infection after exposure compared with 25\% to 50\% of children infected between the ages of 1 and 5 years, $<10 \%$ of those infected in later childhood, and $<5 \%$ of those infected in adulthood. The mechanism for immune tolerance in early infancy is not clearly elucidated but is postulated to be associated with transplacental maternal $\mathrm{HBeAg}$ induction of $\mathrm{T}$ cell tolerance to hepatitis B core antigen ( $\mathrm{HBcAg}$ ) and $\mathrm{HBeAg}$ in the neonate. It has also been noted that neonates infected in utero do not have immunoglobulin $\mathrm{M}$ antibody to $\mathrm{HBcAg}$, manifesting a lack of primary immune response. ${ }^{7,8} \mathrm{HBsAg}$ is not believed to cross the placental barrier but is usually acquired at birth or shortly thereafter. Thus, the presence of HBsAg in cord blood may indicate intrauterine infection. Transplacental transmission has been shown to be

Abbreviations: FDA, Food and Drug Administration; HBCAg, hepatitis B core antigen; HBeAg, hepatitis B e antigen; HBlg, hepatitis B immunoglobulin; HBSAg, hepatitis B surface antigen; HBV, hepatitis B virus; HIV, human immunodeficiency virus.

From the Cedars Sinai Medical Center, Los Angeles, CA

Potential conflict of interest: Nothing to report.

View this article online at wileyonlinelibrary.com

(C) 2013 by the American Association for the Study of Liver Diseases

doi: 10.1002/cld.159 
significantly associated with maternal HBeAg positivity, HBsAg titers, HBV DNA level, and a history of threatened preterm labor. ${ }^{9}$ Studies have examined other risk factors for in utero transmission, and some indicate increased risk with threatened abortion, ${ }^{10}$ while amniocentesis did not appear to increase risk of acquiring HB infection. ${ }^{11}$

The role of maternal viremia has been examined extensively as a significant factor in perinatal transmission. In an early study, Burk et al. ${ }^{12}$ evaluated the role of maternal HBV DNA in a nested case-control study cohort of 773 HBsAgpositive Taiwanese women and found a significant relationship between serum HBV DNA levels and a persistently infected infant. The odds ratio of persistent infant infection was 147 in those born to HBeAg-positive mothers with serum HBV DNA levels $\geq 1.4 \mathrm{ng} / \mathrm{mL}$.

More recent studies have confirmed the important role of HBV DNA in perinatal transmission with varying thresholds for risk. More recently, Wiseman et al. ${ }^{13}$ reported on 313 HBsAg-positive pregnant women with 138 babies born to the HBV DNA-positive women ( $n=213$ ) who were followed for 9 months postpartum. They found a transmission rate of $9 \%$, even with appropriate immunoprophylaxis. All cases of transmission occurred in those with HBV DNA levels $>10^{8}$ copies $/ \mathrm{mL}$. Another retrospective study by Zou et al. ${ }^{14}$ stratifying predelivery DNA with transmission reported $0 \%$ transmission with $<10^{6}$ copies $/ \mathrm{mL}, 3 \% 10^{6}$ $10^{7}$ copies $/ \mathrm{mL}, 6.7 \% 10^{7}-10^{8}$ copies $/ \mathrm{mL}$, and $7.6 \% \geq 10^{8}$ copies/mL.

Studies assessing the infection rate in infants born via cesarean section versus vaginal delivery have not been conclusive in showing a significant difference in neonatal HBV infection, and insufficient data exist to recommend changes in the mode of delivery for HBV-infected women. ${ }^{15}$ Postpartum management should focus on the administration of HBIg and hepatitis B vaccine to the neonate within 12 hours, as the efficacy of HBIg declines as the time between birth and the initial dose increases. The hepatitis $B$ vaccine series should then be continued with two additional doses (a total of three doses: one given at birth, one at 4 weeks to 2 months of age, and one at 6 months of age), and follow-up HBsAg and antibody to HBsAg titers should be tested at 9 to 15 months of age. This passive-active immunoprophylaxis regimen is associated with successful prevention of neonatal infection in $95 \%$ to $100 \%$ of newborns, although the effective implementation of this program varies in some countries and health care systems.

An additional clinical concern often raised in early infancy is the risk of HBV transmission with breastfeeding, as several small early studies reported small amounts of HBsAg in breast milk. More recent, large prospective studies, however, have reported low rates of $\mathrm{HBV}$ transmission in the setting of vaccine prophylaxis. ${ }^{16}$ Current recommendations from the
Centers for Disease Control and Prevention support breastfeeding in HBV-infected women.

\section{Antiviral Therapy in Women of Childbearing Age}

A young woman who is infected with HBV presents myriad clinical dilemmas. If a woman of childbearing age presents with chronic HBV infection and is currently not pregnant, the first issue at hand will be to determine the state of her disease and the necessity of therapy, along with the consideration of her family planning status.

Many young women of childbearing age will be in the immune tolerant stage of disease with HBsAg positivity, HBeAg positivity, and very high levels of viremia with normal serum alanine aminotransferase levels. According to the American Association for the Study of Liver Diseases practice guidelines, ${ }^{17}$ this individual would not be a candidate for antiviral therapy for herself but may be a candidate for antiviral therapy to reduce perinatal transmission late in pregnancy. If this young woman is planning a pregnancy in the distant future, consideration for interferon therapy can be discussed, because it is a defined treatment course of 48 weeks; however, the likelihood of response to interferon is low in persons with immune tolerance.

If the woman is planning a pregnancy in the near future, it is unlikely that a clinician would initiate therapy at this time point due to a paucity of safety data regarding exposure to antiviral therapy during early pregnancy (i.e., the first trimester) in the hepatitis B population. Nucleos(t)ide antiviral therapy has the potential for mitochochondrial toxicity and is of unclear benefit in the young patient with benign liver disease. If the patient is suspected of having more advanced fibrosis/cirrhosis or highly active disease, then the clinical risk benefit assessment may favor initiating therapy and continuing therapy throughout the course of pregnancy (Fig. 1).

If a woman with known chronic HBV infection who is already on antiviral therapy presents with an unexpected pregnancy, the decision of whether to stop therapy, continue therapy, or switch agents is a complex one. If she presents early in the first trimester and has no significant underlying liver disease, stopping therapy with close monitoring may be an option. However, if she presents later in pregnancy (i.e., the second or third trimester) and had high viremia at baseline before initiating therapy, the expectation would be that discontinuation of therapy would result in viral rebound to her previous levels, necessitating discussion of antiviral therapy for reducing perinatal HBV transmission anyway, so continuing therapy would be considered (Fig. 2).

If a woman is newly diagnosed with HBV in the first trimester screening of pregnancy, assessment of her disease status is key. If the patient has previously unrecognized 
Woman of childbearing age presenting with $\mathrm{HBV}$

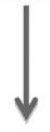

Evidence of advanced fibrosis/cirrhosis?

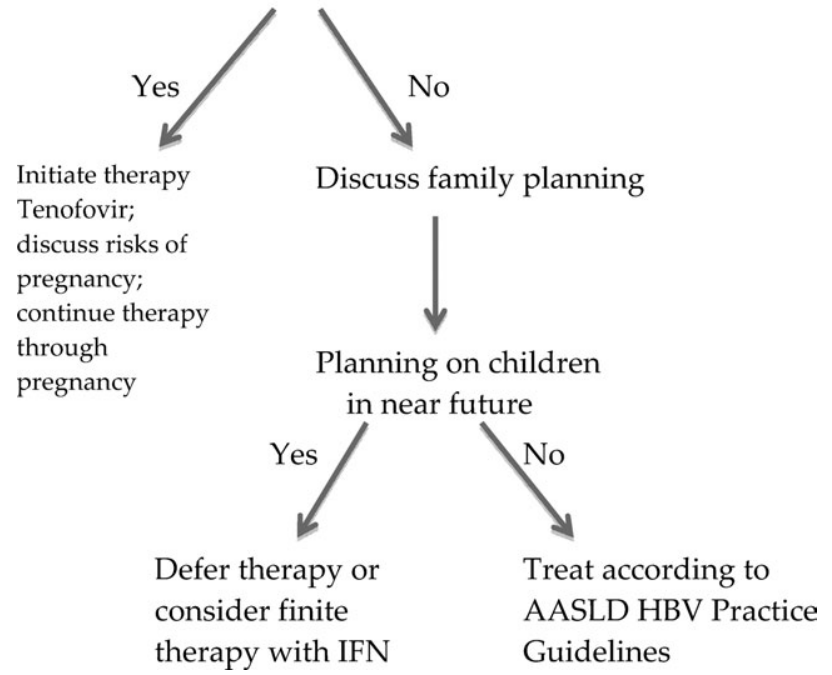

FIGURE 1. Treatment algorithm for an HBV-infected woman who is planning a pregnancy. advanced disease, then initiating therapy may be warranted, and accepting the unknown risk of antiviral therapy may outweigh the risk of decompensation during pregnancy. If, as is more likely the case, the patient has immune tolerant or early disease, then consideration of treatment in the third trimester would be indicated, depending on her risk factors (Fig. 3).

\section{Treatment in the Third Trimester}

If the mother's disease state was stable, not advanced and the clinician had deferred antiviral therapy on the mother's behalf, then as the third trimester approaches, consideration of the perinatal hepatitis B risk to the newborn now becomes the issue. Quantifying the HBV DNA in late second trimester will allow ample time for discussion about the risks and benefits of initiating antiviral therapy. Xu et al. ${ }^{18}$ published a randomized controlled trial of lamivudine versus placebo in highly viremic, HBeAg-positive, HBV-infected pregnant women. At 1-year follow-up, they showed a benefit in mother-child pairs who had received therapy compared with those who had received placebo; however, due to a high number of infants lost to follow-up, especially in the placebo arm, the data were inconclusive. ${ }^{18}$ Meta-analyses on lamivudine treatment to prevent perinatal transmission have been

Woman ALREADY on HBV therapy now gets pregnant

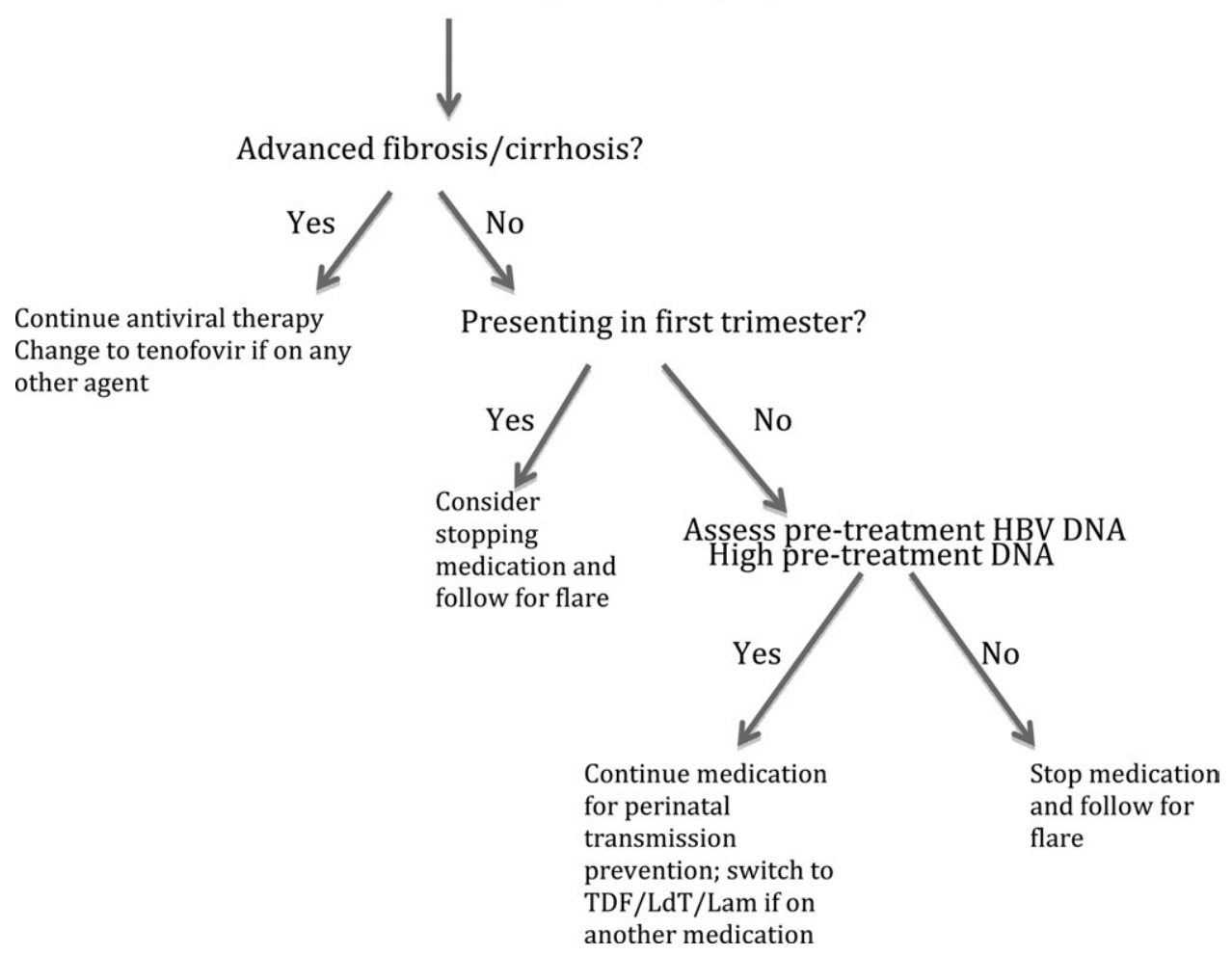

FIGURE 2. Treatment algorithm for an HBV-infected woman who is already on antiviral therapy and presents with an unexpected pregnancy. 


\section{Algorithm for Management of HBV Infection During Pregnancy}

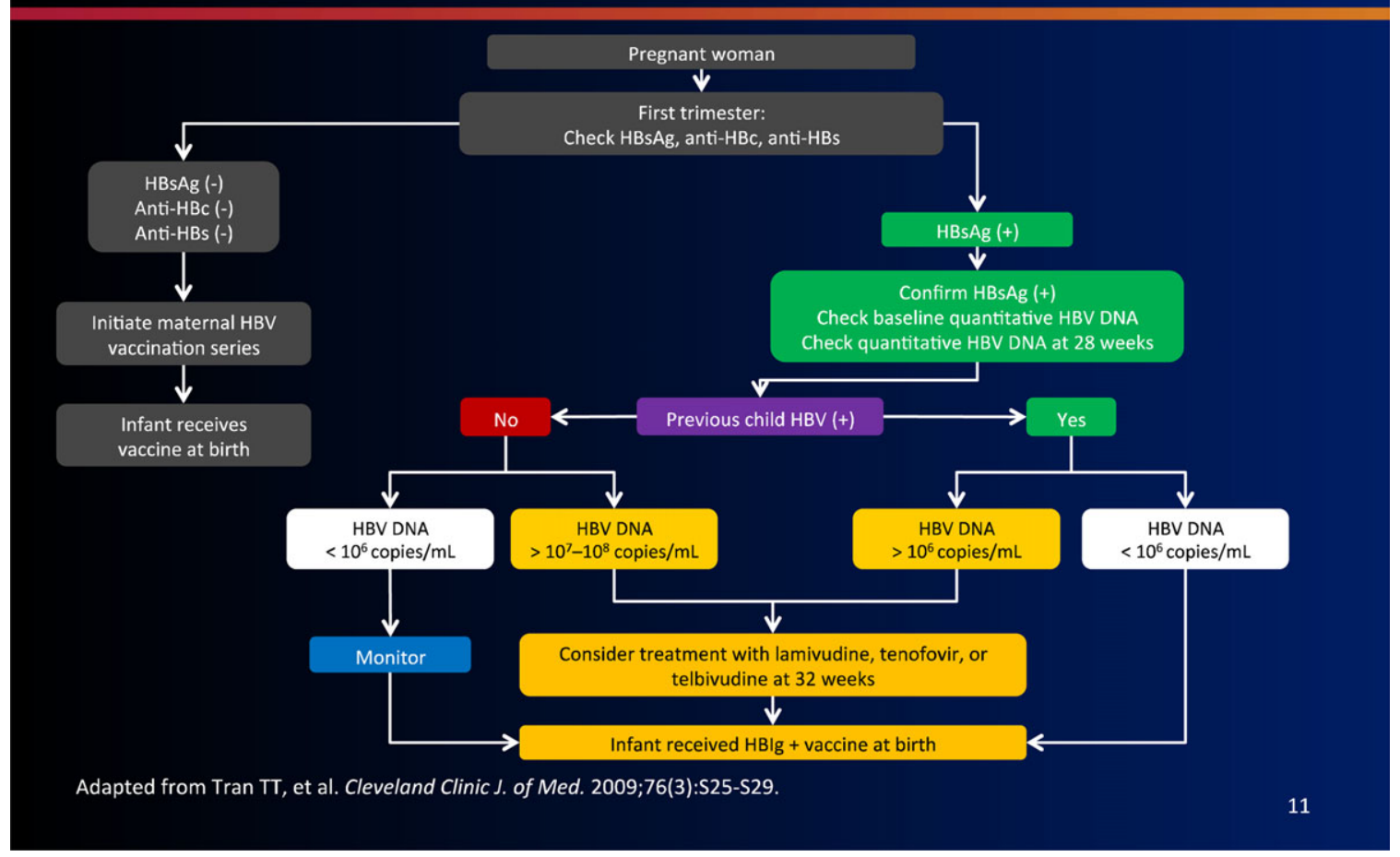

FIGURE 3. Treatment algorithm for the management of HBV infection during pregnancy.

limited by the quality of studies and variability of definitions. $^{19}$

Currently, there are only seven antiviral therapies that have been approved by the US Food and Drug Administration (FDA) for the treatment of HBV. Interferon, both standard and pegylated, is contraindicated in pregnancy due to concerns about interferon-antiproliferative effects on the developing fetus. Of the oral antivirals lamivudine, adefovir, entecavir, telbivudine, and tenofovir, only two are FDA pregnancy class B (telbivudine, tenofovir); the others are all pregnancy class $C$. The oral agent with the most human safety data is lamivudine, due to its use in human immunodeficiency virus (HIV) infection; however, its FDA classification, concerns about development of viral resistance, and its lower potency may reduce its clinical use for some patients in this setting.

Several reports have been published on the use in China of telbivudine to prevent perinatal transmission. In the largest of these studies, Han et al. $^{20}$ reported on 229 HBVinfected pregnant women with high $\mathrm{HBV}$ viral load $\left(>10^{7}\right.$ $\log$ copies $/ \mathrm{mL}$ ) who were treated with telbivudine starting at weeks 20 to 32 of pregnancy and stopped either postnatally (in those who were immune tolerant) or continued (in those who had immune active disease) compared with nonrandomized untreated controls. All infants received HBIg and vaccination as appropriate per protocol and were followed up at 7 months. Perinatal transmission occurred in $8 \%$ of those who were not treated compared with $0 \%$ who did receive telbivudine. No adverse events were noted with telbivudine. $^{20}$

Limited data exist on the treatment of HBV in pregnancy with tenofovir, but extensive safety data in HIV-treated individuals reported in the Antiretroviral Pregnancy Registry ${ }^{21}$ suggest no increase in risk from the general population for birth defects, though these data are limited by the selfreported nature of the registry.

The duration of antiviral therapy when used in the setting of pregnancy is based on the rationale of the initial treatment decision. If a clinician started treatment based on advanced disease in the mother or other maternal issues, then continuing therapy during pregnancy and post-partum would be beneficial to prevent disease progression and flares. Breastfeeding while on therapy is of unknown risk and current labeling guidelines discourage use during breastfeeding. If 
treatment was initiated in order solely to prevent perinatal transmission in a mother with high viremia, once the infant is born and receives the initial HBIg and vaccine, antiviral therapy is of no clear benefit to the infant or the mother. Discontinuing antiviral medication at any point during or after pregnancy requires close monitoring, as immunologic changes in pregnancy have been reported with the potential for flares. 22,23

Thus, in the third trimester, based on accumulating data, if maternal viral HBV DNA levels are $>10^{7} \log$ copies $/ \mathrm{mL}$ (or the equivalent $\mathrm{IU} / \mathrm{mL}$ ), then treatment later in pregnancy can be supported after extensive discussion with the mother

\section{References}

1. World Health Organization. Hepatitis B: fact sheet no. 204. http://who.int/ mediacentre/factsheets/fs204/en/index.html. Accessed December 1, 2012.

2. Kowdley KV, Wang CC, Welch S, Roberts H, Brosgart CL. Prevalence of chronic hepatitis B among foreign born persons living in the United States by country of origin. Hepatology 2012;56:422-433.

3. Stevens CE, Beasley RP, Tsui J, Lee WC. Vertical transmission of hepatitis B antigen in Taiwan. N Engl J Med 1975, 292:771-774.

4. Lee SD, Lo KJ, Tsai YT, Wu JC. Maternal hepatitis B virus DNA in motherinfant transmission. Lancet 1989;1:719.

5. Ngui SL, O'Connell S, Eglin RP, Heptonstall J, Teo CG. Low detection rate and maternal provenance of hepatitis B virus $S$ gene mutants in cases of failed postnatal immunoprophylaxis in England and Wales. J Infect Dis 1997;176: 1360-1365.

6. Karthigesu VD, Allison LM, Ferguson M, Howard CR. A hepatitis B virus variant found in the sera of immunised children induces a conformational change in the HBsAg "a" determinant. J Med Virol 1999;58:346-352.

7. Lin HH, Lee TY, Chen DS, Sung JL, Ohto H, Etoh T, et al. Transplacental leakage of HBeAg-positive maternal blood as the most likely route in causing intrauterine infection with hepatitis B virus. J Pediatr 1987;111:877-881.

8. Lazizi Y, Badur S, Perk Y, Ilter O, Pillot J. Selective unresponsiveness to HBsAg vaccine in newborns related with an in utero passage of hepatitis B virus DNA. Vaccine 1997;15:1095-1100.

9. Xu DZ, Yan YP, Choi BC, Xu JQ, Men K, Zhang JX, et al. Risk factors and mechanism of transplacental transmission of hepatitis B virus: a case-control study. J Med Virol 2002;67:20-26.

10. Lin HH, Lee TY, Chen DS, Sung JL, Ohto H, Etoh T, et al. Transplacental leakage of HBeAg-positive maternal blood as the most likely route in causing intrauterine infection with hepatitis B virus. J Pediatr 1987;111:877-881.

11. Towers CV, Asrat T, Rumney P. The presence of hepatitis B surface antigen and deoxyribonucleic acid in amniotic fluid and cord blood. Am J Obstet Gynecol 2001;184:1514-1518. regarding the risks and benefits. Perinatal transmission at lower levels of HBV DNA $\left(10^{6} \log\right.$ copies $\left./ \mathrm{mL}\right)$ has been shown to be a modest risk (3\%), but this would then need to be weighed against the risk of medication-related birth defects (2\%-3\%). Treatment at levels $<10^{6} \log$ copies $/ \mathrm{mL}$ should be considered as part of the management of advanced disease in the expectant mother, but not for prevention of perinatal transmission.

\section{CORRESPONDENCE}

Tram T. Tran, Medical Director, Liver Transplantation, Cedars Sinai Medical Center, 8635 W. $3^{\text {rd }}$ Street, Suite 590W, Los Angeles, CA 90048. E-mail: trant@cshs.org

12. Burk RD, Hwang LY, Ho GY, Shafritz DA, Beasley RP. Outcome of perinatal hepatitis B virus exposure is dependent on maternal virus load. J Infect Dis 1994;170:1418-1423.

13. Wiseman E, Fraser MA, Holden S, Glass A, Kidson BL, Heron LG, et al. Perinatal transmission of hepatitis B virus: an Australian experience. Med J Aust 2009; 109:489-492.

14. Zou H, Chen Y, Duan Z et al. A retrospective study for clinical outcome of caesarean section on perinatal transmission of hepatitis B virus in infants born to HBeAg positive mothers with chronic hepatitis. Hepatology 2010;52:235A.

15. Lee SD, Lo KJ, Tsai YT, Wu JC, Wu TC, Yang ZL, et al. Role of caesarean section in prevention of mother-infant transmission of hepatitis B. Lancet 1988;2:833-834.

16. Hill JB, Sheffield JS, Kim MJ, Alexander JM, Sercely B, Wendel GD. Risk of hepatitis B transmission in breast-fed infants of chronic hepatitis B carriers. Obstet Gynecol 2002;99:1049-1052.

17. Lok AS, McMahon BJ. Chronic hepatitis B: update 2009. Hepatology 2009;50: $1-36$.

18. Xu WM, Cui YT, Wang L, Yang H, Liang ZQ, Li XM, et al. Lamivudine in late pregnancy to prevent perinatal transmission of hepatitis B virus infection: a multicentre, randomized, double-blind, placebo-controlled study. J Viral Hepat 2009; 16:94-103.

19. Han L, Zhang H-W, Xie J-X, Zhang Q, Wang HY, Cao GW. A meta-analysis of lamivudine for interruption of mother-to-child transmission of hepatitis B virus. World J Gastroenterol 2011;17:4321-4333.

20. Han G-R, Cao M-K, Zhao W, Jiang HX, Wang CM, Bai SF, et al. A prospective and open label study for the efficacy and safety of telbivudine in pregnancy for the prevention of perinatal transmission of hepatitis B virus infection. J Hepatology 2011;55:1215-1221.

21. The Antiretroviral Pregancy Registry Interim Report for 1 January 1989 through 31 July 2011. www.apregistry.com. Accessed December 1, 2012.

22. ter Borg MJ, Leemans WF, de Man RA, Janssen HLA. Exacerbation of chronic hepatitis B infection after delivery. J Vir Hepat 2008;15:37-41.

23. Singhal A, Kanagala R, Jalil S, Wright HI, Kohli V. Chronic HBV with pregnancy: reactivation flare causing fulminant hepatic failure. Ann Hepatol 2011;10:233-236. 